CLINICAL STUDY

\title{
Serum uric acid and its association with metabolic syndrome and carotid atherosclerosis in obese children
}

\author{
Lucia Pacifico $^{1}$, Vito Cantisani ${ }^{2}$, Caterina Anania ${ }^{1}$, Elisabetta Bonaiuto ${ }^{1}$, Francesco Martino ${ }^{1}$, Roberto Pascone ${ }^{3}$ \\ and Claudio Chiesa ${ }^{1,4}$ \\ Departments of ${ }^{1}$ Pediatrics, ${ }^{2}$ Radiological Sciences and ${ }^{3}$ Laboratory Medicine, La Sapienza University of Rome, Viale Regina Elena, 32400161 Rome, Italy \\ and ${ }^{4}$ Molecular Medicine, National Research Council, 00133 Rome, Italy
}

(Correspondence should be addressed to L Pacifico; Email: lucia.pacifico@uniroma1.it)

\begin{abstract}
Objective: The association between hyperuricemia, metabolic syndrome (MS), and atherosclerotic vascular disease has been reported in adults, but very little is known about this association in children. The aims of our study were to ascertain the correlates of uric acid (UA) in a sample of obese children, and to investigate whether UA is associated with carotid intima-media thickness (IMT) independently from classical risk factors including MS.

Methods: We analyzed carotid IMT along with serum triglycerides, total and high-density lipoprotein cholesterol, glucose, insulin, insulin resistance index (as homeostasis model assessment of insulin resistance), alanine aminotransferase, $\gamma$-glutamyltransferase, creatinine, and UA in 120 obese children and 50 healthy control children.

Results: UA concentrations were significantly higher in obese children compared with controls; moreover, they correlated with the most established cardiovascular risk factors. In the group of obese children, after adjustment for age, sex, pubertal stage, and creatinine, an independent association between UA levels and the presence of MS syndrome was observed (unstandardized coefficient, 0.044 (95\% confidence intervals $(\mathrm{CI}) 0.015-0.072) ; P<0.01)$. Carotid IMT significantly increased in the fourth quartile of UA compared with that in the first, second, and third quartile (0.49 (0.46-0.53), $0.53(0.49-0.56)$, and $0.55(0.52-0.59)$ vs 0.61 (95\% CI, $0.58-0.64) ; P<0.01)$. When multivariate analysis was performed after adjusting for age, gender, pubertal stage, creatinine, and MS (considered as a single clinical entity), or the individual components of MS simultaneously included, the association between UA and carotid IMT was significant $(P<0.01)$.

Conclusions: In obese children and adolescents, increased UA levels are associated with carotid atherosclerosis.
\end{abstract}

European Journal of Endocrinology $16045-52$

\section{Introduction}

Several epidemiological studies have shown that hyperuricemia is a risk factor for cardiovascular diseases (CVDs) in the general population (1-3). The contribution of serum uric acid (UA) to atherosclerotic vascular disease, however, remains controversial (4). Some studies argue that the observed association between UA and atherosclerotic vascular disease is attributable to an indirect association of hyperuricemia with cardiovascular risk factors or clustering of these metabolic and hemodynamic risk factors, designated 'metabolic syndrome' (MS) (5, 6). Recent evidence suggests that UA stimulates vascular smooth muscle proliferation and induces endothelial dysfunction. UA has been shown to decrease endothelial nitric oxide production and to lead to endothelial dysfunction and insulin resistance $(7,8)$. Consequently, UA induces vascular inflammation and artery damage, which in turn leads to an increased risk of atherosclerosis. Although many studies in adults have focused on the relationship between UA, MS, and carotid atherosclerosis (9-12), very little is known about this association in children and adolescents. Because atherosclerosis often begins in childhood or young adulthood (13), studies in this age group are important. One additional advantage of examining children is that a diminished potential confusion exists with adult-onset complications. Previous cross-sectional data have shown a close relationship between UA concentrations and cardiovascular risk factors in obese children and adolescents (14-17); however, the role of UA as an independent risk factor for CVD remains to be clarified in children.

Carotid intima-media thickness (IMT) measured noninvasively by ultrasonography is a well-established index of atherosclerosis and directly associated with increased risk of CVD $(18,19)$. This study aimed first to ascertain the correlates of UA in a sample of obese 
children and adolescents, and then to investigate whether UA is associated with carotid IMT independently of classical risk factors including MS.

\section{Methods}

\section{Study subjects}

Between May 2007 and April 2008, 120 children and adolescents with primary obesity (body mass index (BMI) equal to or higher than the age- and sex-specific 97th percentile, which defines obesity according to the Italian BMI charts $(20,21))$ were consecutively enrolled at the Department of Pediatrics, La Sapienza University of Rome, Italy. Exclusion criteria included the presence of renal disease; type 1 or 2 diabetes; any condition known to influence body composition, insulin action, or insulin secretion (e.g. glucocorticoid therapy, hypothyroidism, Cushing's disease); a history of preexisting heart disease; history of use of medications that would affect carotid IMT or lipid profile; and history of alcohol consumption and smoking (where appropriate). All patients underwent physical examination (including measurements of weight, standing height, BMI, and determination of the stage of puberty according to the criteria of Tanner, laboratory tests, and carotid ultrasound (22)). The degree of obesity was quantified using Cole's least mean-square method, which normalizes the skewed distribution of BMI and expresses BMI as a SDS. This measure gives age- and sex-specific estimates of the distribution median, the variation coefficient, and the degree of skew by a maximumlikelihood fitting technique (23). Systolic and diastolic blood pressures (BP) were measured twice at the right arm after a 10-min rest in the supine position by using an automated oscillatory system (Dinamap Vital Signs Monitor, Model 1846 SX; Criticon Incorporated, Tampa, FL, USA).

Over the same study period, 50 healthy children with BMI appropriate for gender and age were recruited to the study if parents consented to participate in the study, which included anthropometric measurements, laboratory investigations, and carotid ultrasound. No control child had a history of alcohol consumption and smoking (where appropriate). Yet, none of the healthy children had family risk factors for premature atherosclerosis, including hyperlipidemia, hypertension, diabetes mellitus, and CVDs. The study was approved by the Hospital Ethics Committee, and informed consent was obtained from subjects' parents prior to assessment.

\section{Laboratory data}

Blood samples were taken from each subject after an overnight fast, for estimation of serum concentrations of glucose, insulin, alanine aminotransferase (ALT), $\gamma$-glutamyltransferase (GGT), total and high-density lipoprotein (HDL) cholesterol, creatinine, and UA. Insulin concentrations were determined using a RIA with polyclonal antibodies (CIS Bio International, Schering S.A., Gif-Sur-Yvette Cedex, France; detection limit, $2.0 \mathrm{mU} / \mathrm{l}$; inter- and intra-assay CVs, $6.4-8.8$ and $4.2-8.2 \%$ respectively) (22). We measured the remaining analytes on a COBAS INTEGRA 800 analyzer (Roche Diagnostics). UA, total cholesterol, HDL cholesterol, triglyceride, and GGT concentrations were assessed with the cassettes COBAS INTEGRA UA ver.2 (detection limit, $0.012 \mathrm{mmol} / \mathrm{l}$; inter- and intra-assay CVs, $1.8-1.9$ and $1.0-1.1 \%$ respectively), total cholesterol ver.2, HDL cholesterol ver.3, triglyceride according to IFCC, and GGT ver.2 respectively (Roche Diagnostics), by enzymatic colorimetric methods; ALT concentration with the cassette ALT according to IFCC by enzymatic u.v. method; glucose concentration with the cassette ver. 3 by a hexokinase method; and creatinine concentration with the cassette creatinine Jaffé by a colorimetric method.

\section{Carotid ultrasound}

High-resolution B-mode ultrasonography of the common carotid arteries was performed with an Aplio XV (Toshiba America Medical Systems, Tustin, CA, USA) with a linear $14 \mathrm{MHz}$ linear transducer, following a standardized protocol (22). In brief, on a longitudinal, two-dimensional ultrasound image of the carotid artery, the posterior (far) wall of the carotid artery was displayed as two bright white lines separated by a hypoechogenic space. The distance between the leading edge of the first bright line of the far wall and the leading edge of the second bright line indicated the carotid artery IMT. The measurement was performed at the common carotid artery near the bifurcation during end diastole. We measured four values on each side, and maximum and mean IMT were calculated separately for each side.

The intraobserver variability of ultrasonographic measurements was $<3 \%$ and all examinations were performed by a single experienced examiner who was blinded to the clinical and biochemical data.

\section{Definitions}

Identification of MS among children is often based on the adult criteria defined by the National Cholesterol Education Program's Adult Treatment Panel (24) and the World Health Organization (25). In the adult definition, a minimum of three of five major criteria (obesity determined by waist circumference, hypertension, low HDL levels, elevated triglyceride levels, and glucose intolerance) should be fulfilled. These criteria have been modified for children $(14,21,26)$. Obesity was defined as a BMI $\geq 97$ th percentile adjusted for age and sex $(20,21)$; hypertriglyceridemia as triglycerides $>95$ th percentile for age and gender (27); low HDL 
cholesterol as concentrations $<5$ th percentile for age and sex (27); elevated BP as systolic or diastolic BP $>$ 95 th percentile for age and sex (28). Impaired fasting glucose was defined according to the criteria of the American Diabetes Association (29). Insulin resistance was determined by a homeostasis model assessment of insulin resistance (HOMA-IR). Scores were calculated as the product of the fasting serum insulin level $(\mathrm{mU} / \mathrm{l})$ and the fasting serum glucose level $(\mathrm{mmol} / \mathrm{l})$, divided by 22.5. HOMA-IR changes during childhood depending on the age, gender, and pubertal stage (30). We have, therefore considered HOMA-IR values above the 95th percentile as an indicator of insulin resistance. Thus, we defined MS in the presence of $\geq 3$ of the following criteria: obesity, hypertension, low HDL cholesterol, elevated triglycerides, and impaired fasting glucose and/or insulin resistance.

\section{Statistical analysis}

Statistical analyses were performed using the SPSS package. Data are expressed either as frequencies or as means with 95\% confidence intervals (CI). Distributions of continuous variables were examined for skewness and kurtosis and were logarithmically transformed, when appropriate. Geometric means are reported for triglycerides, insulin, and HOMA-IR values. Differences between groups were tested for significance using unpaired $t$-test (two-tailed). The $\chi^{2}$ test and Fisher's exact test were used, when appropriate, to compare prevalences. Pearson's correlation and linear regression coefficients were used to examine the relationship between variables, both in the whole population and, separately in controls and in obese children. The association between UA concentrations and the components of the MS were examined by multiple linear regression analysis. The fully adjusted regression model included age, gender, pubertal stage, creatinine, and the individual components of MS entered as continuous variables. The independence of the association of UA with carotid IMT was assessed by multiple linear regression analysis (when the dependent variable, i.e., carotid IMT, was entered as a continuous variable) or logistic (when carotid IMT was modeled as categorical variable and obese subjects were stratified into two groups according to the median value of IMT $(0.55 \mathrm{~mm}))$ regression analyses. In the fully adjusted regression model, together with UA, age, gender, pubertal stage, creatinine, and MS (considered as a single clinical entity) were included as covariates. Separate regression models were also tested with the individual components of MS that were simultaneously included as either continuous or categorical variables in the same equation.

We took the maximum value of carotid IMT as the dependent variable in the regression models because the strongest association between the different measurements of IMT and coronary risk factors in otherwise healthy individuals is achieved by applying the maximum value of IMT and not the mean value of IMT (22). $P<0.05$ was considered to be statistically significant.

\section{Results}

\section{Characteristics of the study population}

The baseline clinical and metabolic characteristics for the 120 obese children and 50 healthy controls are shown in Table 1. Within the entire study population, there was no family history of symptomatic hyperuricemia. Values for glucose, insulin, HOMA-IR, triglycerides, ALT, GGT, creatinine, UA, and BP were significantly higher in the obese children, whereas HDL cholesterol levels were lower. The prevalence of MS in obese subjects was $27.5 \%$ (27.2\% among males and $27.7 \%$ among females).

Table 1 Baseline anthropometric and metabolic characteristics of the study cohort.

\begin{tabular}{|c|c|c|c|}
\hline & $\begin{array}{l}\text { Obese children } \\
\qquad(n=120)\end{array}$ & $\begin{array}{l}\text { Controls } \\
(n=50)\end{array}$ & $\boldsymbol{P}$ \\
\hline Age (years) & $10.6(10.0-11.2)$ & $10.2(9.2-11.2)$ & 0.44 \\
\hline \multicolumn{4}{|l|}{ Sex no. (\%) } \\
\hline Male & $66(55)$ & $23(46)$ & 0.28 \\
\hline \multicolumn{4}{|c|}{ Tanner stage no. (\%) } \\
\hline I & $43(35.8)$ & $21(42)$ & 0.68 \\
\hline II-III & 46 (38.3) & 16 (32) & \\
\hline IV & $31(25.8)$ & $13(26)$ & \\
\hline BMI $\left(\mathrm{kg} / \mathrm{m}^{2}\right)$ & $25.1(24.5-25.7)$ & $17.8(17.0-18.5)$ & $<0.0001$ \\
\hline BMI-SDS & $2.0(1.84-2.1)$ & $0.94(-0.15-0.34)$ & $<0.0001$ \\
\hline $\begin{array}{r}\text { Systolic BP } \\
(\mathrm{mmHg})\end{array}$ & $112(110-114)$ & $100(94-106)$ & $<0.0001$ \\
\hline $\begin{array}{l}\text { Diastolic BP } \\
\qquad(\mathrm{mmHg})\end{array}$ & $71(69-72)$ & $66(62-70)$ & 0.048 \\
\hline $\begin{array}{l}\text { Alanine amino- } \\
\quad \text { transferase }(\mathrm{U} / \mathrm{l})\end{array}$ & $38(32-44)$ & $16(15-18)$ & $<0.0001$ \\
\hline $\begin{array}{l}\gamma \text {-Glutamyl- } \\
\quad \text { transferase }(\mathrm{U} / \mathrm{I})\end{array}$ & $18(17-21)$ & $13(12-14)$ & 0.001 \\
\hline $\begin{array}{l}\text { Total choles- } \\
\text { terol }(\mathrm{mg} / \mathrm{dl})\end{array}$ & $160(151-170)$ & $149(139-160)$ & 0.18 \\
\hline $\begin{array}{l}\text { HDL cholesterol } \\
\text { (mg/dl) }\end{array}$ & $42(40-44)$ & $46(43-49)$ & 0.021 \\
\hline $\begin{array}{l}\text { Triglycerides } \\
\text { (mg/dl) }\end{array}$ & $121(103-139)$ & $72(65-81)$ & 0.004 \\
\hline $\begin{array}{l}\text { Fasting glucose } \\
\qquad(\mathrm{mmol} / \mathrm{l})\end{array}$ & $4.81(4.73-4.89)$ & $4.56(4.43-4.69)$ & 0.001 \\
\hline $\begin{array}{l}\text { Uric acid } \\
\qquad(\mathrm{mmol} / \mathrm{l})\end{array}$ & $0.28(0.27-0.30)$ & $0.19(0.17-0.20)^{\mathrm{a}}$ & $<0.0001$ \\
\hline $\begin{array}{l}\text { Creatinine } \\
\qquad(\mathrm{mmol} / \mathrm{l})\end{array}$ & $54(51-56)$ & $31(29-34)$ & $<0.0001$ \\
\hline Insulin (mU/l) & $17.4(14.6-20.2)$ & $7.38(6.61-8.67)$ & $<0.0001$ \\
\hline $\begin{array}{l}\text { HOMA-IR } \\
\text { values }\end{array}$ & $3.97(3.30-4.64)$ & $1.57(1.35-1.82)$ & $<0.0001$ \\
\hline
\end{tabular}

Results are expressed as $n(\%)$, mean $(95 \% \mathrm{Cl})$, or geometric mean $(95 \% \mathrm{Cl})$ for log-transformed variables.

${ }^{a} U A$ values are within the recently established age- and sex-specific pediatric reference intervals for UA (31). 


\section{UA levels in relation to clinical, anthropo- metric, biochemical parameters, and MS}

Within the entire study population, UA concentrations were significantly correlated with the most established cardiovascular risk factors (Table 2). When the association was restricted to the group of obese subjects, UA levels were significantly associated with BMI-SDS, systolic BP, triglycerides, HDL cholesterol, ALT, GGT, creatinine, insulin, and HOMA-IR, after adjustment for age, gender, and pubertal stage. No correlation was found between UA and total cholesterol. By contrast, when the association was restricted to the control group, UA levels were significantly correlated with BMISDS only (Table 2).

The mean concentrations of UA were significantly higher in the obese patients with MS compared with those without $(0.32(95 \%$ CI, $0.30-0.34)$ vs 0.27 (0.26-0.29); $P<0.0001)$, and they increased with the number of the components of MS (0.26 (95\% CI, 0.24$0.28)$ vs $0.28(0.26-0.30)$ vs $0.31(0.28-0.33)$ vs 0.36 $(0.29-0.43))$ in subjects with $1,2,3$, and $\geq 4$ components of the MS, $P<0.0001$ for trend; Fig. 1). In the group of obese children, an independent association between the concentrations of UA and the presence of MS syndrome (considered as a single clinical entity) was observed (unstandardized regression coefficient, 0.044 (95\% CI, 0.015-0.072); $P<0.01)$ ), after adjustment for age, sex, Tanner stage, and creatinine. When all components of the MS (i.e. BMI-SDS, BP, triglycerides, HDL cholesterol, glucose, and HOMA-IR values) were simultaneously included as continuous variables, UA values were significantly associated with BMI-SDS (unstandardized regression coefficient, 0.042 (0.014-0.071); $P<0.01)$, with systolic BP $(0.002$ (0.0001-0.003); $P<0.05)$, and with HOMA-IR (0.005 (0.002-0.009); $P<0.01)$, after adjustment for age, sex, Tanner stage, and creatinine.

When the obese children were classified into four groups by UA quartiles, it was observed that the

Table 2 Age-, sex-, and Tanner stage-adjusted correlation coefficients between serum uric acid concentration and variables measured in the study participants.

\begin{tabular}{lccc}
\hline & $\begin{array}{c}\text { All cases } \\
(n=170)\end{array}$ & $\begin{array}{c}\text { Controls } \\
(n=50)\end{array}$ & $\begin{array}{c}\text { Obese } \\
(n=120)\end{array}$ \\
\hline BMI-SDS & $0.597^{\S}$ & $0.491^{\S}$ & $0.341^{\S}$ \\
Systolic BP (mmHg) & $0.394^{\S}$ & 0.140 & $0.354^{\S}$ \\
Diastolic BP (mmHg) & $0.218^{\dagger}$ & 0.103 & 0.086 \\
Triglycerides (mg/dl) & $0.292^{\S}$ & 0.127 & $0.211^{*}$ \\
Total cholesterol (mg/dl) & $0.169^{*}$ & 0.146 & 0.158 \\
HDL (mg/dl) & $-0.199^{*}$ & -0.012 & $-0.194^{*}$ \\
Fasting glucose (mmol/l) & 0.113 & 0.134 & 0.028 \\
Insulin (mU/l) & $0.400^{\S}$ & 0.068 & $0.383^{\S}$ \\
HOMA-IR values & $0.430^{\S}$ & 0.098 & $0.409^{\S}$ \\
ALT (U/l) & $0.427^{\S}$ & 0.039 & $0.301^{\ddagger}$ \\
GGT (U/l) & $0.325^{\S}$ & 0.172 & $0.265^{\dagger}$ \\
Creatinine (mmol/l) & $0.485^{\S}$ & 0.196 & $0.240^{\dagger}$ \\
\hline
\end{tabular}

${ }^{\star} P<0.05 ;{ }^{\dagger} P<0.01 ;{ }^{\ddagger} P<0.001 ;{ }^{\S} P<0.0001$.
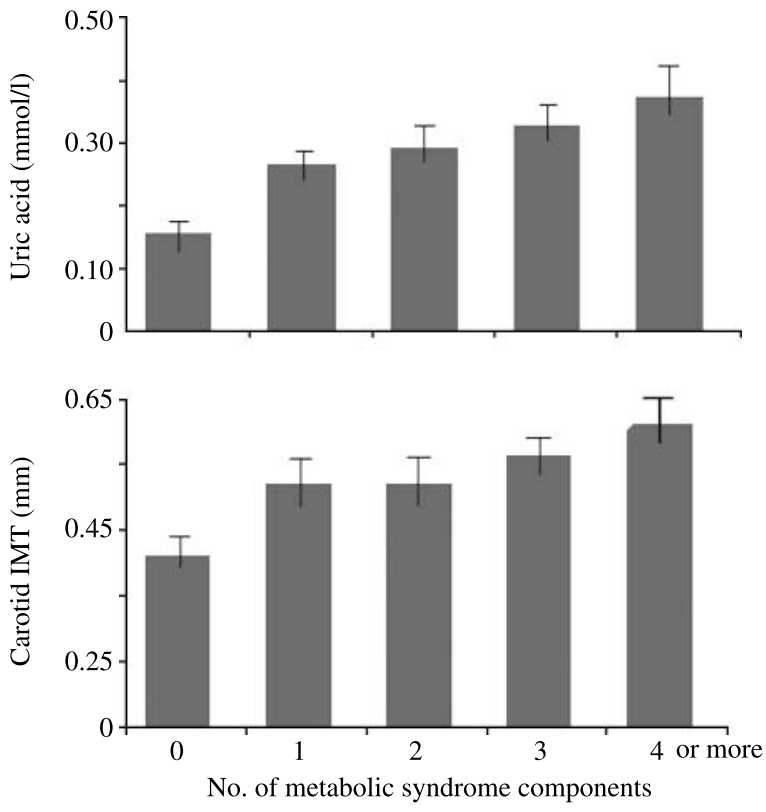

Figure 1 Mean concentrations $(95 \% \mathrm{Cl})$ of serum uric acid as well as of carotid IMT among 170 children and adolescents, by the number of metabolic syndrome components.

prevalence of the MS rose from $4.6 \%$ among obese subjects with concentrations of UA in the first quartile to $17.6,37.9$, and $41.6 \%$ among those with UA levels in the second, third, and fourth quartile respectively $(P<0.01)$.

\section{Carotid IMT in relation to clinical, anthropo- metric, biochemical parameters, and MS}

Compared with controls, obese children showed increased mean carotid IMT $(0.49$ (95\% CI, 0.47$0.51)$ vs 0.40 (95\% CI, $0.38-0.42) \mathrm{mm} ; P<0.0001)$ and maximum $(0.55$ (95\% CI, 0.53-0.57) vs $0.42(95 \%$ CI, 0.39-0.44) mm; $P<0.0001)$. Within the entire study population, carotid IMT were significantly correlated with BMI-SDS, systolic BP, triglycerides, ALT, GGT, glucose, insulin, HOMA-IR, and UA $(r=$ $0.562, P<0.0001)$, after adjustment for age, gender, and pubertal stage (Table 3 ). In the obese children, a significant correlation remained between carotid IMT values and systolic BP, ALT, insulin, HOMA-IR, and UA. By contrast, in the control group no associations were observed between carotid IMT values and systolic BP, ALT, insulin, and HOMA-IR, although a trend was observed between carotid IMT values and UA $(r=$ 0.268, $P=0.069$; Table 3).

Maximum carotid IMT was significantly higher in the obese patients with MS compared with those without (mean, 0.60 (95\% CI, 0.57-0.63) vs 0.53 (0.50-0.55); $P<0.0001)$; in particular, it was 0.53 (95\% CI, 0.50$0.55), 0.53(0.50-0.55), 0.59(0.55-0.62)$, and 0.64 $(0.58-0.71)$ in subjects with $1,2,3$, and $\geq 4$ 
Table 3 Age-, sex-, and Tanner stage-adjusted correlation coefficients between carotid intima-media thickness (IMT) and variables measured in the study participants.

\begin{tabular}{lccc}
\hline & $\begin{array}{c}\text { All cases } \\
(n=170)\end{array}$ & $\begin{array}{c}\text { Controls } \\
(n=50)\end{array}$ & $\begin{array}{r}\text { Obese } \\
(n=120)\end{array}$ \\
\hline BMI-SDS & $0.468^{\S}$ & 0.095 & 0.102 \\
Systolic BP (mmHg) & $0.312^{\S}$ & 0.095 & $0.191^{*}$ \\
Diastolic BP (mmHg) & 0.140 & 0.075 & 0.003 \\
Triglycerides (mg/dl) & $0.165^{\star}$ & 0.134 & 0.027 \\
Total cholesterol (mg/dl) & 0.038 & 0.076 & 0.012 \\
HDL (mg/dl) & -0.091 & -0.037 & -0.001 \\
Fasting glucose (mmol/l) & $0.167^{\star}$ & 0.064 & 0.054 \\
Insulin (mU/l) & $0.348^{\S}$ & 0.100 & $0.251^{\dagger}$ \\
HOMA-IR values & $0.390^{\S}$ & 0.061 & $0.299^{\dagger}$ \\
ALT (U/l) & $0.370^{\S}$ & 0.106 & $0.287^{\dagger}$ \\
GGT (U/l) & $0.241^{\S}$ & 0.029 & 0.165 \\
Uric acid (mmol/l) & $0.562^{\S}$ & $0.268^{\|}$ & $0.390^{\S}$ \\
& & &
\end{tabular}

${ }^{*} P<0.05 ;{ }^{\dagger} P<0.01 ;{ }^{\S} P<0.0001 ;{ }^{\prime \prime} P=0.069$.

components of the MS, $P<0.05$ for trend (Fig. 1). In the group of obese subjects, an independent association between carotid IMT and the presence of MS was observed (unstandardized regression coefficient, 0.064 (95\% CI, 0.026-0.102); $P<0.01$ ), after adjustment for age, sex, and pubertal stage. When the obese children were classified into four groups by UA quartiles, carotid IMT significantly increased in the fourth quartile of UA compared with that in the first, second, and third quartile (0.49 (0.46-0.53), $0.53(0.49-0.56)$, and 0.55 $(0.52-0.59)$ vs 0.61 (95\% CI, $0.58-0.64) ; P<0.01$ for trend); moreover, if patients with and without MS were analyzed separately, carotid IMT was still significantly increased in the fourth quartile of UA (Table 4).

\section{Multiple regression analysis of the association between UA, MS, and carotid IMT in obese children}

When multivariate linear regression analysis was performed, after adjusting for age, gender, pubertal stage, creatinine, and MS (considered as a single clinical entity), the association between UA (entered as a continuous variable) and carotid IMT was statistically significant (unstandardized coefficient $(95 \%$ CI), 0.495

Table 4 Maximum carotid intima-media thickness (IMT) in obese children with and without metabolic syndrome by uric acid quartiles.

\begin{tabular}{|c|c|c|c|}
\hline \multirow[b]{2}{*}{ Quartiles } & \multirow[b]{2}{*}{$\begin{array}{c}\text { Uric acid } \\
(\mathrm{mmol} / \mathrm{l}) \text { mean } \\
(95 \% \mathrm{Cl})\end{array}$} & \multicolumn{2}{|c|}{ Carotid IMT $(\mathrm{mm})$ mean $(95 \% \mathrm{Cl})$} \\
\hline & & $\begin{array}{l}\text { Obese children } \\
\text { with metabolic } \\
\text { syndrome }\end{array}$ & $\begin{array}{l}\text { Obese children } \\
\text { without meta- } \\
\text { bolic syndrome }\end{array}$ \\
\hline 1 & $0.19(0.17-0.21)$ & $-^{\mathrm{a}}$ & $0.49(0.45-0.53)$ \\
\hline 2 & $0.24(0.23-0.25)$ & $0.55(0.48-0.62)$ & $0.51(0.47-0.56)$ \\
\hline 3 & $0.30(0.29-0.31)$ & $0.57(0.52-0.62)$ & $0.52(0.48-0.57)$ \\
\hline 4 & $0.38(0.37-0.39)$ & $0.64(0.61-0.68)^{*}$ & $0.59(0.55-0.63)^{*, \dagger}$ \\
\hline
\end{tabular}

(0.247-0.743), $P<0.0001)$. Even after adjustment for age, gender, pubertal stage, creatinine, and the individual components of MS (BMI-SDS, BP, HDL cholesterol, triglycerides, fasting glucose, and HOMAIR, included as continuous variables), the association between UA and carotid IMT remained statistically significant $(0.482(0.208-0.755), P<0.01)$. In this model, the regression coefficient $(95 \% \mathrm{CI})$ of other covariates independently associated with carotid IMT were as follows: pubertal stage (0.043 (0.013-0.072), $P<0.01)$ and HOMA-IR (0.006 (0.0001-0.011), $P<0.05)$. Similar results were found when we considered carotid IMT as a categorical variable (on the basis of the median value of IMT, obese children were stratified into two groups: those having IMT equal or more than $0.55 \mathrm{~mm}$ and those having IMT less than $0.55 \mathrm{~mm})$ and performed multivariate logistic regression analyses. In this case, again UA was significantly associated with carotid IMT after adjusting for age, gender, Tanner stage, creatinine, and MS (considered as a single clinical entity) or the individual components of MS simultaneously included (Table 5).

\section{Discussion}

In this study, we have shown that UA concentrations are associated with MS, independent of age, sex, Tanner stage, and degree of obesity in obese children and adolescents. We have also demonstrated for the first

Table 5 Multivariate logistic analyses of the association between serum uric acid and maximum carotid intima-media thickness $(\mathrm{IMT})^{\mathrm{a}}$ in obese children.

\begin{tabular}{lccc}
\hline & OR & 95\% CI & $\boldsymbol{P}$ \\
\hline Model 1 & & & \\
Age (years) & 0.707 & $0.469-1.065$ & 0.097 \\
Gender & 0.484 & $0.206-1.137$ & 0.096 \\
Tanner stage & 2.338 & $1.069-5.112$ & 0.033 \\
Uric acid,c & 4.755 & $1.746-12.94$ & 0.002 \\
Creatinine & 0.978 & $0.943-1.013$ & 0.217 \\
Metabolic syndrome & 4.306 & $1.531-12.11$ & 0.006 \\
Model 2 & & & \\
Age (years) & 0.631 & $0.391-1.020$ & 0.060 \\
Gender & 0.566 & $0.228-1.404$ & 0.220 \\
Tanner stage & 2.498 & $1.082-5.766$ & 0.032 \\
Uric acid & 5.451 & $1.951-15.23$ & 0.001 \\
Creatinine & 0.977 & $0.940-1.060$ & 0.250 \\
BMl-SDS & 0.479 & $0.165-1.387$ & 0.175 \\
High blood pressure & 7.109 & $0.474-10.67$ & 0.156 \\
High triglycerides & 1.483 & $0.598-3.678$ & 0.396 \\
Low HDL & 1.299 & $0.534-3.157$ & 0.564 \\
High glucose and/or & 4.888 & $1.006-20.76$ & 0.040 \\
$\quad$ HOMA-IR & & & \\
\hline
\end{tabular}

${ }^{\mathrm{a}}$ Carotid IMT was modeled as categorical variable stratifying obese subjects into two groups according to the median value observed (i.e. $0.55 \mathrm{~mm}$ ).

${ }^{b}$ As carotid IMT significantly increased in the fourth quartile of uric acid compared with that in the other three quartiles, uric acid was modeled as categorical variable (and subjects were stratified into those belonging to the fourth quartile versus those belonging to the other three quartiles).

'When uric acid was also entered in the models as continuous variable, the association was still significant $(P<0.01)$. 
time that UA values are associated with carotid atherosclerosis independent of other atherogenic risk factors in this population.

Previous studies have shown a close relation between hyperuricemia and the MS both in adults and children $(9,11,14,16,21,32,33)$. The prevalence of the MS among our obese children in the lowest quartile of concentrations of UA was very low (4.6\%), while a high percentage $(41.6 \%)$ of the children in the highest quartile of concentrations of UA had the MS. Therefore, our results are consistent with those of Ford et al. who previously demonstrated the association of the prevalence of MS in obese adolescents with quartiles of serum UA concentrations in a nationally representative sample of 1370 males and females aged $12-17$ years (14). In addition, the UA concentration increased monotomically with the number of MS components. Similar findings have also been previously reported by Ford et al. (14).

In children, hyperuricemia has been associated with many of the established risk factors for CVD, including underlying abnormalities of insulin metabolism (i.e. impaired glucose tolerance, insulin resistance, or multiple MS, hypertension, dyslipidemia, obesity, and central adiposity $(16,21,33))$. In the study by Ford et al. abdominal obesity, hypertriglyceridemia, and hyperglycemia remained significantly associated with concentrations of UA even after adjustment for age, sex, race or ethnicity, concentrations of C-reactive protein, and other components of the MS (14). In addition, the association between high BP and concentrations of UA was of borderline significance (14). In a recent study involving the enrollment of 125 consecutive children for the evaluation of hypertension, Feig \& Johnson have shown a continuous and strong correlation of UA with systolic BP in control subjects and in subjects with primary hypertension that was not explained by obesity or decreased renal function (34). Furthermore, the recent observation that allopurinol lowers UA and BP values in adolescents with essential hypertension is of great interest (35). In the present study, we found that concentrations of serum UA were significantly associated with all individual components of the MS after adjustment for age, sex, and Tanner stage. However, when all components of the MS were simultaneously included in the analysis, UA values remained significantly associated with BMI-SDS, with systolic BP, and with HOMA-IR, after adjustment for age, sex, Tanner stage, and creatinine. The strong association in our obese children between UA and BMI-SDS confirm previous studies reporting significant associations between UA concentrations and various anthropometric measures in children and adolescents $(14,16)$. Yet, our finding of a strong association of UA with systolic BP is consistent with a growing literature on the possible role of UA in the pathogenesis of childhood primary hypertension (34-36). Finally, from our data it is suggested that in children, such as in adults (9), hyperuricemia may be a marker for insulin resistance, an underlying condition of the MS (37). Several studies have revealed that insulin resistance is inversely correlated to 24-h urinary UA clearance (38). Therefore, one potential pathophysiologic mechanism linking hyperuricemia with insulin resistance and/or hyperinsulinemia is a decreased renal excretion of UA (4). In fact, insulin enhances renal tubular sodium and urate reabsorption in humans (4). Interestingly, amelioration of insulin resistance by diet or insulin-sensitizing agent has been reported to decrease the serum UA level (39), which may support the notion that hyperuricemia may be a part of insulin resistance syndrome (40). Because insulin resistance and MS are risk factors for carotid atherosclerosis (6), we may have to take these conditions into account when assessing the independence of the relationship between UA and atherosclerosis. In the present study, we showed that the observed link between UA and carotid IMT was truly independent, remaining significant even after adjustment for the degree of insulin resistance.

In recent years, increased attention has been focused on the fact that prediction of atherosclerosis has been more important because developments in preventive medicine have been presenting new treatment modalities. The assessment of carotid IMT is postulated as a surrogate marker of generalized atherosclerosis $(18,19)$.

Several proatherogenic properties have been attributed to UA including activation of endothelial cells (8), platelet activation, and increased platelet adhesiveness $(4,7)$. UA promotes vascular smooth muscle proliferation and upregulates the expression of platelet-derived growth factor (41) and monocyte chemoattractant protein 1 (42). UA has also been shown to stimulate the production of interleukin $1 \beta$, interleukin 6 , tumor necrosis factor- $\alpha$ by human mononuclear cells, and C-reactive protein by cultured human vascular cells (8). Previous studies in adults have investigated the association between UA and carotid atherosclerosis and reported that this may not be independent of other cardiovascular risk factors (9-12). A number of studies have also shown that UA concentrations are influenced by conditions more commonly found in adults, such as impaired renal function, use of diuretics, alcohol consumption, smoking habits, diabetes mellitus, and CVD $(9,11,43,44)$.

In children, to our knowledge, at the time of this writing, only Schiel et al. have previously investigated the association of UA with IMT (17). These authors evaluated 81 overweight or obese children and adolescents (but no controls), and found an association between UA and IMT that was no longer significant when included in a very large regression model (21 variables for 81 participants) with multicollinearity problems. Differences in the compositions of the populations studied, sample size, and statistical adjustment for confounding variables may all have contributed to the conflicting conclusions between that study 
(17) and ours. Thus, our finding of an independent and strong association between UA and carotid IMT among obese children and adolescents, even after adjustment for the MS (considered as a single clinical entity) or its individual components is novel, and because of the absence of chronic factors that confound studies among adults, it may contribute to a better understanding of the link between childhood obesity and adult CVD and have implications for lifestyle and health risks in a juvenile group. As for the adults, obesity in children is in rapid expansion across the world with the potential of adding considerably to the future health burden of cardiovascular and metabolic diseases. Coronary risk factors measured in children are associated with the early development of coronary artery calcifications, and obesity in adolescence is related with a significantly increased risk of early death from coronary heart disease during adulthood (45). For these reasons, the recognition of high-risk subjects is of great importance from a clinical and public health perspective. Measurement of UA is easy in terms of preanalytics, can be performed with simple methods in routine laboratories, and is inexpensive. Thus, a preventive, cost-effective approach may be available with potential implications for public health.

In conclusion, the strong association in obese children between UA and carotid atherosclerosis, independent of classical risk factors, insulin resistance, and components of the MS, supports the notion of a possible role for UA as a risk factor for early signs of atherosclerosis.

\section{Declaration of interest}

The authors declare that there is no conflict of interest that could be perceived as prejudicing the impartiality of the research reported.

\section{Funding}

This research did not receive any specific grant from any funding agency in the public, commercial or not-for-profit sector.

\section{References}

1 Fang J \& Alderman MH. Serum uric acid and cardiovascular mortality: the NHANES I Epidemiologic Follow-up Study, 19711992. National Health and Nutrition Examination Survey. Journal of the American Medical Association 2000283 2404-2410.

2 Bos MJ. Koudstaall PJ. Hofman A, Witteman JC \& Breteler MM. Uric acid is a risk factor for myocardial infarction and stroke: the Rotterdam Study. Stroke 200637 1503-1507.

3 Strasak A, Ruttmann E, Brant L, Kelleher C, Klenk J, Concin H, Diem G, Pfeiffer K, Ulmer H \& the VHM \& PP Study Group. Serum uric acid and risk of cardiovascular mortality: a prospective longterm study of 83,683 Austrian men. Clinical Chemistry $2008 \mathbf{5 4}$ 273-284.

4 Lippi G, Montagnana M, Franchini M, Favaloro EJ \& Targher G. The paradoxical relationship between serum uric acid and cardiovascular disease. Clinica Chimica Acta 2008392 1-7.
5 Nagahama K, Isei K, Inoue T, Touna T, Ikemiya Y \& Takishita S. Hyperuricemia and cardiovascular risk factor clustering in a screened cohort in Okinawa, Japan. Hypertension Research 2004 27 227-233.

6 Bonora E, Kiechl S, Willeit J, Oberhollenzer F, Egger G, Bonadonna RC \& Muggeo M. Bruneck study. Carotid atherosclerosis and coronary heart disease in the metabolic syndrome: prospective data from the Bruneck study. Diabetes Care 200326 1251-1257.

7 Johnson RJ, Kang DH, Feig D, Kivlighn S, Kanellis J, Watanabe S, Tuttle KR, Rodriguez-Iturbe B, Herrera-Acosta J \& Mazzali M. Is there a pathogenetic role for uric acid in hypertension and cardiovascular and renal disease? Hypertension $2003 \mathbf{4 1}$ $1183-1190$

8 Kanellis J \& Kang DH. Uric acid as a mediator of endothelial dysfunction, inflammation, and vascular disease. Seminars in Nephrology 200525 39-42.

9 Ishizaka N, Ishizaka Y, Toda E-I, Nagai R \& Yamakado M. Association between serum uric acid, metabolic syndrome, and carotid atherosclerosis in Japanese individuals. Arteriosclerosis, Thrombosis, and Vascular Biology 200525 1038-1044.

10 Ishizaka N, Ishizaka Y, Toda E-I, Hashimoto H, Nagai R \& Yamakado M. Higher serum uric acid is associated with increased arterial stiffness in Japanese individuals. Atherosclerosis 2007192 131-137.

11 Kawamoto R, Tomita H, Oka Y \& Ohtsuka N. Relationship between serum uric acid concentration, metabolic syndrome and carotid atherosclerosis. Internal Medicine 200645 605-614.

12 Iribarren C, Folsom AR, Eckfeldt JH, McGovern PG \& Nieto FJ. Correlates of uric acid and its association with asymptomatic carotid atherosclerosis: the ARIC Study. Atherosclerosis Risk in Communities. Annals of Epidemiology 19966 331-340.

13 Berenson GS, Srinivasan SR, Bao W, Newman WP III, Tracy RE \& Wattigney WA. Association between multiple cardiovascular risk factors and atherosclerosis in children and young adults. New England Journal of Medicine 1998338 1650-1656.

14 Ford ES, Li C, Cook S \& Choi HK. Serum concentrations of uric acid and the metabolic syndrome among US children and adolescents. Circulation $20071152526-2532$.

15 Invitti C, Guzzaloni G, Gilardini L, Morabito F \& Viberti G. Prevalence and concomitants of glucose intolerance in European obese children and adolescents. Diabetes Care 200326 118-124.

16 Denzer C, Muche R, Mayer H, Heinze E, Debatin K-M \& Wabitsch M. Serum uric acid levels in obese children and adolescents: linkage to testosterone levels and pre-metabolic syndrome. Journal of Pediatric Endocrinology and Metabolism 2003 16 1225-1232.

17 Schiel R, Beltschikow W, Radon S, Kramer G, Perenthaler T \& Stein G. Increased carotid intima-media thickness and associations with cardiovascular risk factors in obese and overweight children and adolescents. European Journal of Medical Research 200712 503-508.

18 de Groot E, Hovingh GK, Wiegman A, Duriez P, Smit AJ, Fruchart JC \& Kastelein JJ. Measurement of arterial wall thickness as a surrogate marker for atherosclerosis. Circulation 2004109 (Suppl 1) III33-III38.

19 Raitakari OT, Juonala M, Kähönen M, Taittonen L, Laitinen T, Mäki-Torkko N, Järvisalo MJ, Uhari M, Jokinen E, Rönnemaa T, Akerblom HK \& Viikari JS. Cardiovascular risk factors in childhood and carotid artery intima-media thickness in adulthood: the cardiovascular risk in young Finns study. Journal of the American Medical Association $20032902277-2283$.

20 Cacciari E, Milani S, Balsamo A, Dammacco F, De Luca F, Chiarelli F, Pasquino AM, Tonini G \& Vanelli M. Italian crosssectional growth charts for eight, weight and BMI (6-20 y). European Journal of Clinical Nutrition 200256 171-180.

21 Invitti C, Maffeis C, Gilardini L, Pontiggia B, Mazzilli G, Girola A. Sartorio A, Morabito F \& Viberti GC. Metabolic syndrome in obese Caucasian children: prevalence using WHO-derived criteria and association with nontraditional cardiovascular risk factors. International Journal of Obesity 200630 627-633. 
22 Pacifico L, Cantisani V, Ricci P, Osborn JF, Schiavo E, Anania C, Ferrara E, Dvisic G \& Chiesa C. Nonalcoholic fatty liver disease and carotid atherosclerosis in children. Pediatric Research 200863 423-427.

23 Cole TJ, Bellizzi MC, Flegal KM \& Dietz WH. Establishing a standard definition for child overweight and obesity worldwide: international survey. BMJ 2000320 1240-1243.

24 Executive Summary of the Third Report of the National Cholesterol Education Program (NCEP). Expert Panel on detection, evaluation, and treatment of high blood cholesterol in adults (Adult Treatment Panel III). Journal of the American Medical Association 2001285 2486-2497.

25 Alberti KG \& Zimmet PZ. Definition, diagnosis and classification of diabetes mellitus and its complications. 1. Diagnosis and classification of diabetes mellitus provisional report of a WHO consultation. Diabetic Medicine 199815 539-553.

26 Boney CM, Verma A, Tucker R \& Vohr BR. Metabolic syndrome in childhood: association with birth weight, maternal obesity, and gestational diabetes mellitus. Pediatrics 2005115 e290-e296.

27 American Academy of Pediatrics. National Cholesterol Education Program: report of the Expert Panel on blood cholesterol levels in children and adolescents. Pediatrics 199289 525-584.

28 National High Blood Pressure Education Program Working Group on High Blood Pressure in Children and Adolescents. The fourth report on the diagnosis, evaluation, and treatment of high blood pressure in children and adolescents. Pediatrics 2004114 555-576.

29 Expert Committee on the Diagnosis and Classification of Diabetes Mellitus. Report of the Expert Committee on the diagnosis and classification of diabetes mellitus. Diabetes Care 199720 1183-1197.

30 Allard P, Delvin EE, Paradis G, Hanley JA, O'Loughlin J, Lavallée C, Levy E \& Lambert M. Distribution of fasting plasma insulin, free fatty acids, and glucose concentrations and of homeostasis model assessment of insulin resistance in a representative sample of Quebec children and adolescents. Clinical Chemistry 200349 644-649.

31 Pediatric Reference Intervals, edn 6, chapter chemistry tests, pp. 3-215. Eds Soldin SJ, Brugnara C \& Wong EC, Washington, DC; American Association for Clinical Chemistry (AACC), 2007.

32 Nakagawa $\mathrm{T}$, $\mathrm{Hu} \mathrm{H}$, Zharikov $\mathrm{S}$, Tuttle KR, Short RA, Glushakova O, Ouyang X, Feig DI, Block ER, Herrera-Acosta J, Patel JM \& Johnson RJ. A causal role for uric acid in fructoseinduced metabolic syndrome. American Journal of Physiology. Renal Physiology 2006290 F625-F631.

33 Molnar D, Decsi T \& Koletzko B. Reduced antioxidant status in obese children with multimetabolic syndrome. International Journal of Obesity 200428 1197-1202.

34 Feig DI \& Johnson RJ. Hyperuricemia in childhood primary hypertension. Hypertension $2003 \mathbf{4 2} 247-252$.
35 Feig DI, Nakagawa T, Karumanchi SA, Oliver WJ, Kang D-H, Finch J \& Johnson RJ. Hypothesis: uric acid, nephron number, and the pathogenesis of essential hypertension. Kidney International $200466281-287$.

36 Feig DI. Uric acid and hypertension in adolescents. Seminars in Nephrology 200525 32-38.

37 Rathmann W, Funkhouser E, Dyer AR \& Roseman JM. Relations of hyperuricemia with the various components of the insulin resistance syndrome in young black and white adults: the CARDIA study. Coronary Artery Risk Development in Young Adults. Annals of Epidemiology $1998 \mathbf{8} 250-261$.

38 Facchini F, Chen YD, Hollenbeck CB \& Reaven GM. Relationship between resistance to insulin-mediated glucose uptake, urinary uric acid clearance, and plasma uric acid concentration. Journal of the American Medical Association 1991266 3008-3011.

39 Tsunoda S, Kamide K, Minami J \& Kawano Y. Decreases in serum uric acid by amelioration of insulin resistance in overweight hypertensive patients: effect of a low-energy diet and an insulinsensitizing agent. American Journal of Hypertension 200215 697-701.

40 Clausen JO, Borch-Johnsen K, Ibsen H \& Pedersen O. Analysis of the relationship between fasting serum uric acid and the insulin sensitivity index in a population-based sample of 380 young healthy Caucasians. European Journal of Endocrinology 1998138 63-69.

41 Rao GN, Corson MA \& Berk BC. Uric acid stimulates vascular smooth muscle cell proliferation by increasing platelet-derived growth factor A-chain expression. Journal of Biological Chemistry $19912668604-8608$.

42 Kanellis J, Watanabe S, Li JH, Kang DH, Li P, Nakagawa T, Wamsley A, Sheikh-Hamad D, Lan HY, Feng L \& Johnson RJ. Uric acid stimulates monocyte chemoattractant protein-1 production in vascular smooth muscle cells via mitogen-activated protein kinase and cyclooxygenase-2. Hypertension 200341 1287-1293.

43 Maclachlan MJ \& Rodnan GP. Effect of food, fast and alcohol on serum uric acid and acute attacks of gouts. American Journal of Medicine 196742 38-57.

44 Niskanen LK, Laaksonen DE, Nyyssonen K, Alfthan G, Lakka HM, Lakka TA \& Salonen JT. Uric acid level as a risk factor for cardiovascular and all-cause mortality in middle-aged men: a prospective cohort study. Archives of Internal Medicine $2004 \mathbf{1 6 4}$ $1546-1551$.

45 Li S, Chen W, Srinivasan SR, Bond MG, Tang R, Urbina EM \& Berenson GS. Childhood cardiovascular risk factors and carotid vascular changes in adulthood: the Bogalusa Heart Study. Journal of the American Medical Association $20032902271-2276$.

Received 16 October 2008

Accepted 18 October 2008 